\title{
Functional Solutions for a Plane Problem in Magnetohydrodynamics
}

\author{
Giovanni CimatTi
}

ABstRact - We study the flow in a channel of a fluid obeying the equations of magnetohydrodynamics under the hypotheses that viscosity, resistivity and thermal conductivity depend on the temperature. The special class of solutions for which two functional dependences between temperature and magnetic field and velocity and magnetic field exist is considered.

\section{Introduction}

We study in this paper a class of steady, incompressible and rectilinear flows of viscous, electrically and thermally conducting fluids along cylindrical channels of arbitrary cross-section in the framework of the equations of magnetohydrodynamics. The open and bounded subset of $\boldsymbol{R}^{2}$, representing the cross-section of the pipe is referred to the orthogonal frame $O x_{1} x_{2} x_{3} . O x_{3}$ is the axis of the channel with unit vector $\boldsymbol{i}_{3}$. The magnetic field $\boldsymbol{H}$ and the velocity $\boldsymbol{v}$ are assumed to be of the form

$$
\begin{aligned}
\boldsymbol{H} & =h\left(x_{1}, x_{2}\right) \boldsymbol{i}_{3} \\
\boldsymbol{v} & =v\left(x_{1}, x_{2}\right) \boldsymbol{i}_{3} .
\end{aligned}
$$

Moreover, we suppose

$$
p=p\left(x_{1}, x_{2}\right) .
$$

The electrical resistivity $\rho$, the viscosity $v$ and the thermal conductivity $\kappa$ are assumed to be given functions of the temperature $u$ and of $v$ and $h$. The

(*) Indirizzo dell'A.: Department of Mathematics, University of Pisa, Largo Bruno Pontecorvo 5, 56127, Pisa, Italy.

E-mail: cimatti@dm.unipi.it 
domain $\Omega$ is multiply connected with its boundary $\Gamma$ consisting of two disjoint closed curves $\Gamma_{1}$ and $\Gamma_{2}$ of class $C^{1}$. From the general equations of magnetohydrodynamics and from the energy equation [6] we obtain, in view of (1.1) and (1.2) the following boundary value problem $(P)$ [4]. For more details we refer to the Appendix.

$$
\begin{gathered}
\nabla \cdot(\rho(u, v, h) \nabla h)=0 \text { in } \Omega \\
\nabla \cdot(v(u, v, h) \nabla v)=0 \text { in } \Omega \\
\nabla \cdot(\kappa(u, v, h) \nabla u+v(u, v, h) v \nabla v+\rho(u, v, h) h \nabla h)=0 \text { in } \Omega \\
h=0 \text { on } \Gamma_{1}, h=\bar{h} \text { on } \Gamma_{2} \\
v=0 \text { on } \Gamma_{1}, v=\bar{v} \text { on } \Gamma_{2} \\
u=0 \text { on } \Gamma_{1}, u=\bar{u} \text { on } \Gamma_{2},
\end{gathered}
$$

where $\bar{h}, \bar{v}$ and $\bar{u}$ are given positive constants. We prove in Section 2 that if the functions $\kappa, v$ and $\rho$ have the special form (2.9) below, problem $(P)$ has one and only one solution. Section 3 deals with the functional solutions of problem $(P)$ i.e. with solutions for which there exist functional dependences between $u$ and $h$ and between $v$ and $h$. We prove the existence of a one-to-one correspondence between the functional solutions and the solutions of a two-point problem for a system of first order differential equations depending on two parameters. First order ordinary differential equations depending on parameters whose solutions must satisfy two boundary conditions have been considered, among others, in [2], [3], [7] and [8]. In Section 4 a theorem of existence and uniqueness is proved when resistivity, viscosity and thermal conductivity depend only on the temperature.

\section{A result of existence and uniqueness}

To prove the next theorem we need the following elementary

Lemma 1. Let $\hat{\kappa}(u) \in C^{1}([0, \infty)), \hat{v}(v) \in C^{1}([0, \bar{v}]), \in C^{1}([0, \bar{h}])$

$$
\hat{\kappa}(u) \geq \kappa_{0}>0
$$

and

$$
\hat{v}(u) \geq v_{0}>0 .
$$


The transformation

$$
\begin{gathered}
\theta=K(u)+M(v)+\frac{h^{2}}{2} \\
\psi=N(v),
\end{gathered}
$$

defined for $u \in[0, \infty), v \in[0, \bar{h}]$ and depending on the parameter $h \in[0, \bar{h}]$, where

$$
K(u)=\int_{0}^{u} \hat{\kappa}(t) d t, \quad M(v)=\int_{0}^{v} t \hat{v}(t) d t, \quad N(v)=\int_{0}^{v} \hat{v}(t) d t
$$

is globally invertible for every $h \in[0, \bar{h}]$.

Proof. By (2.2), (2.4) has an inverse

$$
v=N^{-1}(\psi)
$$

defined in $[0, N(\bar{v})]$. Substituting (2.5) into (2.3) we obtain

$$
\theta=K(u)+M\left(N^{-1}(\psi)\right)+\frac{h^{2}}{2} .
$$

Since by (2.1) $K(u)$ is strictly increasing and

$$
\lim _{u \rightarrow \infty} K(u)=\infty
$$

(2.6) has, with respect to $u$, a unique solution

$$
u=K^{-1}\left(\theta+M\left(N^{-1}(\psi)\right)+\frac{h^{2}}{2}\right) .
$$

The inverse of (2.3), (2.4) is therefore (2.8), (2.5).

THEOREM 1. If $\kappa$ and $v$ are given by

$$
\kappa(u, v, h)=\rho(u, v, h) \hat{\kappa}(u), v(u, v, h)=\rho(u, v, h) \hat{v}(v),
$$

where $\rho(u, v, h), \hat{\kappa}(u)$ and $\hat{v}(v)$ are $C^{1}$ functions such that

$$
\rho(u, v, h) \geq \rho_{0}>0, \hat{\kappa}(u) \geq \kappa_{0}>0, \hat{v}(v) \geq v_{0}>0
$$

when $u \geq 0, \bar{v} \geq v \geq 0, \bar{h} \geq h \geq 0$, then problem $(P)$ has one and only one solution. 
Proof. By the maximum principle the solutions of problem $(P)$ satisfy

$$
u(\boldsymbol{x}) \geq 0, \bar{v} \geq v(\boldsymbol{x}) \geq 0, \bar{h} \geq h(\boldsymbol{x}) \geq 0 \text { in } \Omega, \quad \boldsymbol{x}=\left(x_{1}, x_{2}\right) .
$$

Define $K(u), M(v)$ and $N(v)$ as in Lemma 1 . The transformation

$$
\begin{gathered}
\theta=K(u)+M(v)+\frac{h^{2}}{2} \\
\psi=N(v)
\end{gathered}
$$

by Lemma 1 is globally invertible with inverse

$$
\begin{gathered}
u=U(\theta, \psi, h)=K^{-1}\left(\theta+M\left(N^{-1}(\psi)\right)+\frac{h^{2}}{2}\right) \\
v=V(\psi)=N^{-1}(\psi) .
\end{gathered}
$$

Define

$$
\alpha(\theta, \psi, h)=\rho(U(\theta, \psi, h), V(\psi), h) .
$$

Let $(u(\boldsymbol{x}), v(\boldsymbol{x}), h(\boldsymbol{x}))$ be any classical solution of problem $(P)$ and set

$$
\begin{gathered}
\theta(\boldsymbol{x})=K(u(\boldsymbol{x}))+M(v(\boldsymbol{x}))+\frac{h(\boldsymbol{x})^{2}}{2} \\
\psi(\boldsymbol{x})=N(v(\boldsymbol{x})) .
\end{gathered}
$$

Problem $(P)$ can be reformulated in terms of $\theta, \psi$ and $h$ as problem $(\mathcal{P})$

$$
\begin{gathered}
\nabla \cdot(\alpha(\theta, \psi, h) \nabla \theta)=0 \text { in } \Omega \\
\nabla \cdot(\alpha(\theta, \psi, h) \nabla \psi)=0 \text { in } \Omega \\
\nabla \cdot(\alpha(\theta, \psi, h) \nabla h)=0 \text { in } \Omega \\
\theta=0 \text { on } \Gamma_{1}, \theta=\bar{\theta}=K(\bar{u})+M(\bar{v})+\frac{\bar{h}^{2}}{2} \text { on } \Gamma_{2} \\
\psi=0 \text { on } \Gamma_{1}, \psi=\bar{\psi}=N(\bar{v}) \text { on } \Gamma_{2} \\
h=0 \text { on } \Gamma_{1}, h=\bar{h} \text { on } \Gamma_{2} .
\end{gathered}
$$

We claim that problem $(\mathcal{P})$ has one and only one solution. For, let $(\theta(\boldsymbol{x}), \psi(\boldsymbol{x}), h(\boldsymbol{x}))$ be any solution of $(\mathcal{P})$. Define

$$
\begin{gathered}
\zeta(\boldsymbol{x})=\theta(\boldsymbol{x})-\frac{\bar{\theta}}{\bar{h}} h(\boldsymbol{x}) \\
\xi(\boldsymbol{x})=\psi(\boldsymbol{x})-\frac{\bar{\psi}}{\bar{h}} h(\boldsymbol{x}) .
\end{gathered}
$$


We have $\zeta=0$ and $\xi=0$ on $\Gamma$. On the other hand, by (2.14) and (2.16)

$$
\nabla \cdot(\alpha(\theta, \psi, h) \nabla \zeta)=\nabla \cdot(\alpha(\theta, \psi, h) \nabla \theta)-\frac{\bar{\theta}}{\bar{h}} \nabla \cdot(\alpha(\theta, \psi, h) \nabla h)=0 \text { in } \Omega
$$

and, by (2.15) and (2.16),

$$
\nabla \cdot(\alpha(\theta, \psi, h) \nabla \xi)=\nabla \cdot(\alpha(\theta, \psi, h) \nabla \psi)-\frac{\bar{\psi}}{\bar{h}} \nabla \cdot(\alpha(\theta, \psi, h) \nabla h)=0 \text { in } \Omega .
$$

Multiplying (2.17) by $\zeta$ and integrating by parts over $\Omega$ we have

$$
\int_{\Omega} \alpha(\theta, \psi, h)|\nabla \zeta|^{2} d x_{1} d x_{2}=0 .
$$

Similarly from (2.18) we obtain

$$
\int_{\Omega} \alpha(\theta, \psi, h)|\nabla \xi|^{2} d x_{1} d x_{2}=0 .
$$

Hence

$$
\zeta(\boldsymbol{x})=0, \xi(\boldsymbol{x})=0 \text { in } \Omega .
$$

Thus $\theta(\boldsymbol{x}), \psi(\boldsymbol{x})$ and $h(\boldsymbol{x})$ are related by the functional relations

$$
\begin{aligned}
\theta(\boldsymbol{x}) & =\frac{\bar{\theta}}{\bar{h}} h(\boldsymbol{x}) \\
\psi(\boldsymbol{x}) & =\frac{\bar{\psi}}{\bar{h}} h(\boldsymbol{x}) .
\end{aligned}
$$

Hence the equation (2.16) can be rewritten

$$
\begin{gathered}
\nabla \cdot\left(\alpha\left(\frac{\bar{\theta}}{\bar{h}} h, \frac{\bar{\psi}}{\bar{h}} h, h\right) \nabla h\right)=0 \\
h=0 \text { on } \Gamma_{1}, h=\bar{h} \text { on } \Gamma_{2} .
\end{gathered}
$$

This problem has one and only one solution. For, let

$$
w=\mathcal{F}(h)=\int_{0}^{h} \alpha\left(\frac{\bar{\theta}}{\bar{h}} t, \overline{\bar{\psi}} \overline{\bar{h}} t, t\right) d t .
$$

Equation (2.21) under this transformation becomes

$$
\Delta w=0 \text { in } \Omega, w=0 \text { on } \Gamma_{1}, w=\mathcal{F}(\bar{h}) \text { on } \Gamma_{2} .
$$


Hence

$$
h(\boldsymbol{x})=\mathcal{F}^{-1}(w(\boldsymbol{x})), \theta(\boldsymbol{x})=\frac{\bar{\theta}}{\bar{h}} \mathcal{F}^{-1}(w(\boldsymbol{x})), \psi(\boldsymbol{x})=\frac{\bar{\psi}}{\bar{h}} \mathcal{F}^{-1}(w(\boldsymbol{x})) .
$$

By (2.11) and (2.12) we obtain as the only solution of problem $(P)$

$$
\begin{gathered}
u(\boldsymbol{x})=K^{-1}\left[\frac{\bar{\theta}}{\bar{h}} \mathcal{F}^{-1}(w(\boldsymbol{x}))+M\left(N^{-1}\left(\frac{\bar{\psi}}{\bar{h}} \mathcal{F}^{-1}(w(\boldsymbol{x}))\right)\right)+\frac{1}{2}\left(\mathcal{F}^{-1}(w(\boldsymbol{x}))\right)^{2}\right] \\
v(\boldsymbol{x})=N^{-1}\left(\frac{\bar{\psi}}{\bar{h}} \mathcal{F}^{-1}(w(\boldsymbol{x}))\right) \\
h(\boldsymbol{x})=\mathcal{F}^{-1}(w(\boldsymbol{x})) .
\end{gathered}
$$

\section{The functional solutions}

The assumptions (2.9) are quite special. To treat more general situations we use a different definition of solutions suggested by the proof of Theorem 1 and in particular by the functional relations (2.19) and (2.20) between $\theta, \psi$ and $h$.

DeFinition. We say that a classical solution $(u(\boldsymbol{x}), v(\boldsymbol{x}), h(\boldsymbol{x}))$ of problem $(P)$ is a functional solution if two functions $\mathcal{U}(h) \in C^{2}([0, \bar{h}])$, $\mathcal{V}(h) \in C^{2}([0, \bar{h}])$ exist such that

$$
u(\boldsymbol{x})=\mathcal{U}(h(\boldsymbol{x})), v(\boldsymbol{x})=\mathcal{V}(h(\boldsymbol{x})) .
$$

THEOREM 2. If

$$
\rho(u, v, h) \geq \rho_{0}>0
$$

then there exists a one-to-one correspondence between the set $\{F S\}$ of the functional solutions of problem $(P)$ and the set $\{T P P\}$ of the solutions $(\mathcal{U}(h), \mathcal{V}(h), \gamma, \mu)$ of the two-point problem $(T P P)$

$$
\begin{gathered}
v(\mathcal{U}, \mathcal{V}, h) \frac{d \mathcal{V}}{d h}=\gamma \rho(\mathcal{U}, \mathcal{V}, h) \\
\kappa(\mathcal{U}, \mathcal{V}, h) \frac{d \mathcal{U}}{d h}+v(\mathcal{U}, \mathcal{V}, h) \mathcal{V} \frac{d \mathcal{V}}{d h}+h \rho(\mathcal{U}, \mathcal{V}, h)=\mu \rho(\mathcal{U}, \mathcal{V}, h) \\
\mathcal{U}(0)=0, \mathcal{U}(\bar{h})=\bar{u} \\
\mathcal{V}(0)=0, \mathcal{V}(\bar{h})=\bar{v} .
\end{gathered}
$$


Proof. We define the map $\mathcal{I}:\{T P P\} \rightarrow\{P\}$ as follows. Suppose $(\mathcal{U}(h), \mathcal{V}(h), \gamma, \mu) \in\{T P P\}$ and consider the nonlinear boundary value problem

$$
\begin{gathered}
\nabla \cdot(\rho(\mathcal{U}(h), \mathcal{V}(h), h) \nabla h)=0 \text { in } \Omega \\
h=0 \text { on } \Gamma_{1}, h=\bar{h} \text { on } \Gamma_{2} .
\end{gathered}
$$

It is easily seen that (3.6), (3.7) has one and only one solution. For, let

$$
w=R(h)=\int_{0}^{h} \rho(\mathcal{U}(t), \mathcal{V}(t), t) d t .
$$

By (3.1) $R$ maps one-to-one $[0, \bar{h}]$ onto $[0, R(\bar{h})]$. Moreover, under (3.8) problem (3.6), (3.7) becomes

$$
\begin{gathered}
\Delta w=0 \text { in } \Omega \\
w=0 \text { on } \Gamma_{1}, w=R(\bar{h}) \text { on } \Gamma_{2} .
\end{gathered}
$$

Problem (3.9), (3.10) has one and only one solution. Therefore, the only solution to problem (3.6), (3.7) is given by $h(\boldsymbol{x})=R^{-1}(w(\boldsymbol{x}))$. We claim that

$$
(u(\boldsymbol{x}), v(\boldsymbol{x}), h(\boldsymbol{x}))=(\mathcal{U}(h(\boldsymbol{x})), \mathcal{V}(h(\boldsymbol{x})), h(\boldsymbol{x})) \in\{P\} .
$$

We have by (3.2) and (3.6)

$$
\begin{aligned}
\nabla \cdot(v(u(\boldsymbol{x}), v(\boldsymbol{x}), h(\boldsymbol{x})) & \nabla v)=\nabla \cdot\left(v(\mathcal{U}(h(\boldsymbol{x})), \mathcal{V}(h(\boldsymbol{x})), h(\boldsymbol{x})) \frac{d \mathcal{V}}{d h} \nabla h\right)= \\
= & \gamma \nabla \cdot(\rho(\mathcal{U}(h(\boldsymbol{x})), \mathcal{V}(h(\boldsymbol{x})), h(\boldsymbol{x})) \nabla h)=0 \text { in } \Omega
\end{aligned}
$$

Moreover, by (3.3) and (3.6)

$$
\begin{array}{r}
\text { (3.13) } \nabla \cdot[\kappa(u, v, h) \nabla u+v v(u, v, h) \nabla v+h \rho(u, v, h) \nabla h]= \\
=\nabla \cdot\left[\left(\kappa(\mathcal{U}(h), \mathcal{V}(h), h) \frac{d \mathcal{U}}{d h}+\mathcal{V}(h) v(\mathcal{U}(h), \mathcal{V}(h), h) \frac{d \mathcal{V}}{d h}+h \rho(\mathcal{U}(h), \mathcal{V}(h), h) \nabla h\right]=\right. \\
=\mu \nabla \cdot(\rho(\mathcal{U}(h), \mathcal{V}(h), h) \nabla h)=0 \text { in } \Omega .
\end{array}
$$

All the boundary conditions of problem $(P)$ are also satisfied in view of (3.4) and (3.5). Hence $\mathcal{I}$ is well-defined. On the other hand, $\mathcal{I}$ is one-to-one. For, let $(\mathcal{U}(h), \mathcal{V}(h), \gamma, \mu) \in\{T P P\},(\tilde{\mathcal{U}}(h), \tilde{\mathcal{V}}(h), \tilde{\gamma}, \tilde{\mu}) \in\{T P P\}$ and $(u(\boldsymbol{x}), v(\boldsymbol{x}), h(\boldsymbol{x}))=$ $\mathcal{I}(\mathcal{U}(h), \mathcal{V}(h), \gamma, \mu),(\tilde{u}(\boldsymbol{x}), \tilde{v}(\boldsymbol{x}), \tilde{h}(\boldsymbol{x}))=\mathcal{I}(\tilde{\mathcal{U}}(h), \tilde{\mathcal{V}}(h), \tilde{\gamma}, \tilde{\mu})$. If $\mathcal{I}(\mathcal{U}(h), \mathcal{V}(h), \gamma, \mu)=$ $\mathcal{I}(\tilde{\mathcal{U}}(h), \tilde{\mathcal{V}}(h), \tilde{\gamma}, \tilde{\mu})$ we have $u(\boldsymbol{x})=\tilde{u}(\boldsymbol{x}), v(\boldsymbol{x})=\tilde{v}(\boldsymbol{x})$ and $h(\boldsymbol{x})=\tilde{h}(\boldsymbol{x})$, but $u(\boldsymbol{x})=\mathcal{U}(h(\boldsymbol{x})), \tilde{u}(\boldsymbol{x})=\tilde{\mathcal{U}}(h(\boldsymbol{x}))$ and $v(\boldsymbol{x})=\mathcal{V}(h(\boldsymbol{x})), \tilde{v}(\boldsymbol{x})=\tilde{\mathcal{V}}(h(\boldsymbol{x}))$. Hence 
$\mathcal{U}(h(\boldsymbol{x}))=\tilde{\mathcal{U}}(\tilde{h}(\boldsymbol{x}))=\tilde{\mathcal{U}}(h(\boldsymbol{x}))$ and $\mathcal{V}(h(\boldsymbol{x}))=\tilde{\mathcal{V}}(\tilde{h}(\boldsymbol{x}))=\tilde{\mathcal{V}}(h(\boldsymbol{x}))$. Thus

$$
\mathcal{U}(h)=\tilde{\mathcal{U}}(h), \mathcal{V}(h)=\tilde{\mathcal{V}}(h) .
$$

This implies also $\gamma=\tilde{\gamma}$ and $\mu=\tilde{\mu}$. Finally $\mathcal{I}$ is surjective. For, let $(u(\boldsymbol{x}), v(\boldsymbol{x}), h(\boldsymbol{x}))=(\mathcal{U}(h(\boldsymbol{x})), \mathcal{V}(h(\boldsymbol{x})), h(\boldsymbol{x})) \in\{F S\}$. Define

$$
\theta=\int_{0}^{h} v(\mathcal{U}(t), \mathcal{V}(t), t) \frac{d \mathcal{V}}{d t}(t) d t
$$

$\psi=\int_{0}^{h}\left(\kappa(\mathcal{U}(t), \mathcal{V}(t), t) \frac{d \mathcal{U}}{d t}(t)+\mathcal{V}(t) v(\mathcal{U}(t), \mathcal{V}(t), t) \frac{d \mathcal{V}}{d t}(t)+t \rho(\mathcal{U}(t), \mathcal{V}(t), t)\right) d t$

$$
\zeta=\int_{0}^{h} \rho(\mathcal{U}(t), \mathcal{V}(t), t) d t
$$

and

$$
\Theta(\boldsymbol{x})=\theta(h(\boldsymbol{x})), \Psi(\boldsymbol{x})=\psi(h(\boldsymbol{x})), Z(\boldsymbol{x})=\zeta(h(\boldsymbol{x})) .
$$

By (1.4), (1.5) and (1.6) we have

$$
\Delta \Theta=0, \Delta \Psi=0, \Delta Z=0 \text { in } \Omega
$$

with

$$
\begin{aligned}
& \Theta=0 \text { on } \Gamma_{1}, \Theta=A \text { on } \Gamma_{2} \\
& \Psi=0 \text { on } \Gamma_{1}, \Psi=B \text { on } \Gamma_{2} \\
& Z=0 \text { on } \Gamma_{1}, Z=C \text { on } \Gamma_{2}
\end{aligned}
$$

where

$$
\begin{gathered}
A=\int_{0}^{\bar{h}} v(\mathcal{U}(t), \mathcal{V}(t), t) \frac{d \mathcal{V}}{d t}(t) d t \\
B=\int_{0}^{\bar{h}}\left[\kappa(\mathcal{U}(t), \mathcal{V}(t), t) \frac{d \mathcal{U}}{d t}(t)+\mathcal{V}(t) v(\mathcal{U}(t), \mathcal{V}(t), t) \frac{d \mathcal{V}}{d t}(t)+t \rho(\mathcal{U}(t), \mathcal{V}(t), t)\right] d t \\
C=\int_{0}^{\bar{h}} \rho(\mathcal{U}(t), \mathcal{V}(t), t) d t
\end{gathered}
$$


and $C \neq 0$ by (3.1). Let $z(\boldsymbol{x})$ be given by the solution of the problem

$$
\Delta z=0 \text { in } \Omega, z=0 \text { on } \Gamma_{1}, z=1 \text { on } \Gamma_{2} .
$$

We have

$$
\Theta(\boldsymbol{x})=A z(\boldsymbol{x}), \Psi(\boldsymbol{x})=B z(\boldsymbol{x}), Z(\boldsymbol{x})=C z(\boldsymbol{x}) .
$$

Setting

$$
\gamma=\frac{A}{C}, \mu=\frac{B}{C}
$$

we obtain

$$
\Theta(\boldsymbol{x})=\gamma Z(\boldsymbol{x}), \Psi(\boldsymbol{x})=\mu Z(\boldsymbol{x})
$$

and by (3.15), (3.16), and (3.17) we have $\theta=\gamma \zeta$ and $\psi=\mu \zeta$ i.e.

$$
\int_{0}^{h} v(\mathcal{U}(t), \mathcal{V}(t), t) \frac{d \mathcal{V}}{d t}(t) d t=\gamma \int_{0}^{h} \rho(\mathcal{U}(t), \mathcal{V}(t), t) d t
$$

$$
\begin{array}{r}
\int_{0}^{h}\left[\kappa(\mathcal{U}(t), \mathcal{V}(t), t) \frac{d \mathcal{U}}{d t}(t)+\mathcal{V}(t) \mathcal{V}(\mathcal{U}(t), \mathcal{V}(t), t) \frac{d \mathcal{V}}{d t}(t)+t \rho(\mathcal{U}(t), \mathcal{V}(t), t)\right] d t= \\
=\mu \int_{0}^{h} \rho(\mathcal{U}(t), \mathcal{V}(t), t) d t
\end{array}
$$

Taking the derivative with respect to $h$ in (3.24) and (3.25) we obtain (3.2) and (3.3). Hence, with $\gamma$ and $\mu$ given by (3.22) we have $\mathcal{I}(\mathcal{U}(h), \mathcal{V}(h), \gamma, \mu)=(u(\boldsymbol{x}), v(\boldsymbol{x}), h(\boldsymbol{x}))$.

As an immediate consequence of Theorem 2 problem $(P)$ has one and only one functional solution if the corresponding problem $(T P P)$ has one and only one solution. Moreover, the search of the functional solution of problem $(P)$ is broken into two steps: (i) to find the solutions $(\mathcal{U}(h), \mathcal{V}(h), \gamma, \mu)$ of problem $(T P P)$ and (ii) to solve problem (3.20). Step (ii) contains, so-to-speak, the geometry of problem $(P)$ and step (i) the nonlinear part.

To give a theorem of existence for the two-point problem (TPP) we note that (3.2), (3.3), (3.4) and (3.5) can be rewritten as follows

$$
\frac{d \mathcal{V}}{d h}=\gamma \frac{\rho(\mathcal{U}, \mathcal{V}, h)}{v(\mathcal{U}, \mathcal{V}, h)}
$$




$$
\begin{gathered}
\frac{d \mathcal{U}}{d h}=(-\gamma \mathcal{V}-h+\mu) \frac{\rho(\mathcal{U}, \mathcal{V}, h)}{\kappa(\mathcal{U}, \mathcal{V}, h)} \\
\mathcal{U}(0)=0, \mathcal{U}(\bar{h})=\bar{u} \\
\mathcal{V}(0)=0, \mathcal{V}(\bar{h})=\bar{v} .
\end{gathered}
$$

THEOREM 3. If

$$
\begin{gathered}
\kappa(u, v, h)>0, \rho(u, v, h)>0, v(u, v, h)>0 \\
\left.K_{2} \geq \frac{\rho(u, v, h)}{\kappa(u, v, h)}\right) \geq K_{1}>0
\end{gathered}
$$

and

$$
\left.N_{2} \geq \frac{\rho(u, v, h)}{v(u, v, h)}\right) \geq N_{1}>0
$$

the two-point problem (3.26)-(3.29) has at least one solution.

Proof. We transform problem (3.26)-(3.29) in a system of two Volterra-Fredholm integral equations. From (3.26) and (3.29) we have

$$
\gamma=\tilde{\gamma}=\frac{\bar{v}}{\int_{0}^{\bar{h}}\left[\frac{\rho}{v}\right] d t}
$$

where

$$
\left[\frac{\rho}{v}\right]=\frac{\rho(\mathcal{U}(t), \mathcal{V}(t), h(t))}{v(\mathcal{U}(t), \mathcal{V}(t), h(t))}
$$

Moreover, from (3.27) and (3.28) we obtain

$$
\mu=\tilde{\mu}=\frac{1}{\int_{0}^{\bar{h}}\left[\frac{\rho}{\kappa}\right] d t}\left(\bar{u}+\tilde{\gamma} \int_{0}^{\bar{h}} \mathcal{V}(t)\left[\frac{\rho}{\kappa}\right] d t+\int_{0}^{\bar{h}} t\left[\frac{\rho}{\kappa}\right] d t\right)
$$

where

$$
\left[\frac{\rho}{\kappa}\right]=\frac{\rho(\mathcal{U}(t), \mathcal{V}(t), h(t))}{\kappa(\mathcal{U}(t), \mathcal{V}(t), h(t))} .
$$

Substituting (3.33) and (3.34) in (3.26) and (3.27) and integrating with re- 
spect to $h$ we have the system of integral equations

$$
\begin{gathered}
\mathcal{U}(h)=\tilde{\mu} \int_{0}^{h}\left[\frac{\rho}{\kappa}\right] d t-\tilde{\gamma} \int_{0}^{h} \mathcal{V}(t)\left[\frac{\rho}{\kappa}\right] d t-\int_{0}^{h} t\left[\frac{\rho}{\kappa}\right] d t \\
\mathcal{V}(h)=\tilde{\gamma} \int_{0}^{h}\left[\frac{\rho}{v}\right] d t .
\end{gathered}
$$

From (3.33) we have

$$
0 \leq \tilde{\gamma} \leq G_{2}=: \frac{\bar{v}}{N_{1}} \bar{h}
$$

and from (3.36) and (3.26)

$$
\begin{gathered}
0 \leq \mathcal{V}(h) \leq V_{2}=: \frac{N_{2}}{N_{1}} \bar{v} \bar{h}^{2} \\
\frac{d \mathcal{V}}{d h}(h) \leq \frac{N_{2}}{N_{1}} \bar{v} \bar{h} .
\end{gathered}
$$

Moreover, by (3.34) and (3.37)

$$
0 \leq \tilde{\mu} \leq M_{2}=: \frac{1}{K_{1} \bar{h}}\left[\bar{u}+\frac{\bar{v} \bar{h}^{3} N_{2} K_{2}}{N_{1}^{2}}+\frac{\bar{h}^{2} K_{2}}{2}\right] .
$$

Hence, by (3.35) and (3.27)

$$
\begin{gathered}
|\mathcal{U}(h)| \leq U_{2}=: M_{2} K_{2} h+\frac{\bar{v}^{2} \bar{h}^{4} K_{2} N_{2}}{N_{1}^{2}}+K^{2} \bar{h}^{2} \\
\left|\frac{d \mathcal{U}}{d h}\right| \leq D_{2}=:\left(V_{2} G_{2}+\bar{h}+M_{2}\right) K_{2} .
\end{gathered}
$$

Using (3.38), (3.39), (3.41) and (3.42) we can apply the Schauder fixed point theorem. For, define

$$
B=\left\{(\mathcal{U}(h), \mathcal{V}(h)) \in C^{0}([0, \bar{h}]) \times C^{0}([0, \bar{h}]) ;|\mathcal{U}(h)| \leq U_{2},|\mathcal{V}(h)| \leq V_{2}\right\}
$$

and let $\mathcal{T}: B \rightarrow C^{0}([0, \bar{h}]) \times C^{0}([0, \bar{h}]), \mathcal{T}=\left(\mathcal{T}_{1}, \mathcal{T}_{2}\right)$

$$
\begin{gathered}
\mathcal{T}_{1}(\mathcal{U}, \mathcal{V})=\tilde{\gamma} \int_{0}^{h}\left[\frac{\rho}{v}\right] d t . \\
\mathcal{T}_{2}(\mathcal{U}, \mathcal{V})=\tilde{\mu} \int_{0}^{h}\left[\frac{\rho}{\kappa}\right] d t-\tilde{\gamma} \int_{0}^{h} \mathcal{V}(t)\left[\frac{\rho}{\kappa}\right] d t-\int_{0}^{h} t\left[\frac{\rho}{\kappa}\right] d t .
\end{gathered}
$$


We have $\mathcal{T}(B) \subseteq B$. Moreover, proceeding as in the proof of (3.39) and (3.42) we find that $\mathcal{T}(B)$ is bounded in $C^{1}([0, \bar{h}]) \times C^{1}([0, \bar{h}])$ and thus compact in $C^{0}([0, \bar{h}]) \times C^{0}([0, \bar{h}])$ by Arzela's theorem. We conclude that the two-point problem (3.26)-(3.29) has at least one solution.

As a consequence of the theorem just proved and of Theorem 1 problem $(P)$ has at least one functional solution if (3.1), (3.30), (3.31) and (3.32) are satisfied.

\section{A result of uniqueness}

In general [5] the two-point problem (TPP) has more than one solution. It is therefore interesting to give conditions under which the solution is unique. For example, if condition (2.9) holds we have as corresponding twopoint problem

$$
\begin{gathered}
\hat{v}(\mathcal{V}) \frac{d \mathcal{V}}{d h}=\gamma \\
\hat{\kappa}(\mathcal{U}) \frac{d \mathcal{U}}{d h}+\mathcal{V} \hat{v}(\mathcal{V}) \frac{d \mathcal{V}}{d h}+h \hat{\rho}(h)=\mu \\
\mathcal{U}(0)=0, \mathcal{U}(\bar{h})=\bar{u} \\
\mathcal{V}(0)=0, \mathcal{V}(\bar{h})=\bar{v}
\end{gathered}
$$

and it is easily seen that in this case the solution is unique. In the next theorem a result of uniqueness is given when $\kappa, v$ and $\rho$ depend only on the temperature $u$. In practical cases the main dependence of $\kappa, v$ and $\rho$ is precisely from the temperature. In the proof we use the following Lemma [1] .

Lemma 2. If $\beta(\theta) \in C^{1}([0, \infty)), \beta(\theta) \geq 0$ and $\beta^{\prime}(\theta) \leq 0$ then the problem

$$
-\frac{d^{2} \theta}{d h^{2}}=1+\gamma^{2} \beta(\theta), \theta(0)=0, \theta(\bar{h})=\bar{\theta}>0
$$

has, for every $\gamma \in \boldsymbol{R}^{1}$, one and only one solution.

Proof. Simply note that $\theta(h) \geq 0$ by the maximum principle and that $\frac{d}{d \theta}\left(1+\gamma^{2} \beta(\theta)\right)=\gamma^{2} \beta^{\prime}(\theta) \leq 0$. 
THEOREM 4. If $\kappa(u), v(u)$ and $\rho(u) \in C^{1}([0, \infty))$ and satisfy

$$
\begin{gathered}
\kappa(u)>0, \rho(u)>0, v(u)>0 \\
\int_{0}^{\infty} \frac{\kappa(t)}{\rho(t)} d t=\infty \\
\rho^{\prime}(u) v(u) \leq \rho(u) v^{\prime}(u)
\end{gathered}
$$

and

$$
\frac{\rho(u)}{v(u)} \geq r_{0}>0
$$

then there exists one and only one functional solution of problem $(P)$.

Proof. According to Theorem 2 the two-point problem corresponding to problem $(P)$ is, in this case:

$$
\begin{gathered}
v(\mathcal{U}) \frac{d \mathcal{V}}{d h}=\gamma \rho(\mathcal{U}) \\
\frac{\kappa(\mathcal{U})}{\rho(\mathcal{U})} \frac{d \mathcal{U}}{d h}+\gamma \mathcal{V}+h=\mu \\
\mathcal{U}(0)=0, \mathcal{U}(\bar{h})=\bar{u} \\
\mathcal{V}(0)=0, \mathcal{V}(\bar{h})=\bar{v} .
\end{gathered}
$$

We note that

$$
\gamma>0
$$

since $\gamma \leq 0$ is incompatible with (4.2) and (4.8). By (4.3) the function $\theta=F(\mathcal{U})$

$$
F(\mathcal{U})=\int_{0}^{\mathcal{U}} \frac{\kappa(t)}{\rho(t)} d t
$$

maps diffeomorphically $[0, \infty)$ onto $[0, \infty)$. The system (4.6)-(4.9) becomes, in terms of $\theta$ and $\mathcal{V}$,

$$
\begin{gathered}
\frac{d \mathcal{V}}{d h}=\gamma \beta(\theta), \mathcal{V}(0)=0, \mathcal{V}(\bar{h})=\bar{v} \\
\frac{d \theta}{d h}+\gamma \mathcal{V}+h=\mu, \theta(0)=0, \theta(\bar{h})=\bar{\theta}=F(\bar{u}),
\end{gathered}
$$


where

$$
\beta(\theta)=\frac{\rho\left(F^{-1}(\theta)\right)}{v\left(F^{-1}(\theta)\right)} .
$$

From (4.13) we have

$$
\frac{d \theta^{2}}{d h^{2}}+\gamma \frac{d \mathcal{V}}{d h}+1=0
$$

Substituting (4.12) into (4.15) we obtain

$$
\begin{aligned}
& -\frac{d \theta^{2}}{d h^{2}}=1+\gamma^{2} \beta(\theta) \\
& \theta(0)=0, \theta(\bar{h})=\bar{\theta} .
\end{aligned}
$$

If (4.4) holds we have $\beta^{\prime}(\theta) \leq 0$. Thus, by Lemma 2 problem (4.16), (4.17) has for every $\gamma$ one and only one solution $\theta(h ; \gamma)$ and

$$
\mathcal{U}(h ; \gamma)=F^{-1}(\theta(h ; \gamma))
$$

Moreover, by (4.12)

$$
\mathcal{V}(h)=\gamma \int_{0}^{h} \beta(\theta(t ; \gamma)) d t .
$$

The parameter $\gamma$, still at our disposal, is now used to satisfy the boundary condition $\mathcal{V}(\bar{h})=\bar{v}$ which, by (4.19), reads

$$
\bar{v}=\gamma \int_{0}^{\bar{h}} \beta(\theta(t ; \gamma)) d t .
$$

To prove that (4.20), as an equation in $\gamma$, has one and only one solution we define

$$
G(\gamma)=\gamma \int_{0}^{\bar{h}} \beta(\theta(t ; \gamma)) d t .
$$

We have $G(0)=0$ and $G(\gamma)>$ for all $\gamma>0$. Moreover,

$$
G^{\prime}(\gamma)=\int_{0}^{\bar{h}} \beta(\theta(t ; \gamma)) d t+\gamma \int_{0}^{\bar{h}} \beta^{\prime}(\theta(t ; \gamma)) \frac{\partial \theta}{\partial \gamma}(t ; \gamma) d t .
$$


We claim that $\frac{\partial \theta}{\partial \gamma}(t ; \gamma) \leq 0$. By the continuous differentiability with respect to the parameter $\gamma$ and by (4.13) we have

$$
\begin{gathered}
\frac{d \theta_{\gamma}}{d h}=\mathcal{V}(h) \\
\theta_{\gamma}(0)=0 .
\end{gathered}
$$

On the other hand, by (4.12) and (4.10) we have $\mathcal{V}(h ; \gamma)>0$. Thus, from (4.22) and (4.23) we obtain

$$
\theta_{\gamma}(h ; \gamma)<0
$$

Hence, by (4.21) and (4.5)

$$
G^{\prime}(\gamma) \geq \int_{0}^{\bar{h}} \beta(\theta(t, \gamma ; \gamma)) d t \geq r_{0} \bar{h}>0 .
$$

Therefore (4.20) has, for every $\bar{v}$, one and only one solution $\tilde{\gamma}$. We conclude that the only solution of problem (4.6)-(4.9) is given by

$$
\begin{gathered}
\mathcal{U}(h)=F^{-1}(\theta(h ; \tilde{\gamma})) \\
\mathcal{V}(h)=\tilde{\gamma} \int_{0}^{h} \beta(\theta(t ; \tilde{\gamma})) d t .
\end{gathered}
$$

By Theorem 2 there is only one functional solution of problem $(P)$ which we obtain in the usual way: we solve the problem

$$
\nabla \cdot(\rho(\mathcal{U}(h)) \nabla h)=0 \text { in } \Omega, h=0 \text { on } \Gamma_{1}, h=\bar{h} \text { on } \Gamma_{2}
$$

defining

$$
w=\mathcal{H}(h)=\int_{0}^{h} \rho(\mathcal{U}(t)) d t
$$

By (4.2) $\mathcal{H}$ maps ono-to-one $[0, \bar{h}]$ onto $[0, \bar{w}]$ where $\bar{w}=\mathcal{H}(\bar{h})$. In term of $w$ problem (4.27) reads

$$
\Delta w=0 \text { in } \Omega, w=0 \text { on } \Gamma_{1}, w=\bar{w} \text { on } \Gamma_{2} .
$$

Let $w(\boldsymbol{x})$ be the solution of (4.28). We have

$$
h(\boldsymbol{x})=\mathcal{H}^{-1}(w(\boldsymbol{x}))
$$


and

$$
\begin{aligned}
& u(\boldsymbol{x})=\mathcal{U}(h(\boldsymbol{x}))=\mathcal{U}\left(\mathcal{H}^{-1}(w(\boldsymbol{x}))\right) \\
& v(\boldsymbol{x})=\mathcal{V}(h(\boldsymbol{x}))=\mathcal{V}\left(\mathcal{H}^{-1}(w(\boldsymbol{x}))\right) .
\end{aligned}
$$

The only functional solution of problem $(P)$ is given by (4.29), (4.30) and (4.31).

We stress the difference between Theorem 1, where we found uniqueness in the general class of classical solutions $\{C S\}$, and Theorem 4 where uniqueness is proved in the smaller class of functional solutions $\{F S\}$. It would be interesting to show that the solution, under the hypotheses of Theorem 4 , is unique also in $\{C S\}$.

\section{Appendix}

We derive here the equations (1.4), (1.5) and (1.6) entering in problem $(P)$. We assume the magnetic permeability $\mu_{0}$ to be a constant. Moreover, the pressure $p$, the magnetic field $\boldsymbol{H}$ and the velocity $\boldsymbol{v}$ are supposed to have the special form (1.1), (1.2) and (1.3). Using the Maxwell equation

$$
\boldsymbol{J}=\frac{c}{4 \pi} \nabla \times \boldsymbol{H}
$$

the magnetohydrodynamic body forces

$$
\boldsymbol{f}=\frac{\mu_{0}}{c} \boldsymbol{J} \times \boldsymbol{H}
$$

by (1.1) take the form

$$
\boldsymbol{f}=-\frac{\mu_{0}}{2 \pi} h \frac{\partial h}{\partial x_{1}} \boldsymbol{i}_{1}-\frac{\mu_{0}}{2 \pi} h \frac{\partial h}{\partial x_{2}} \boldsymbol{i}_{2} .
$$

Thus, by (5.2), (1.2) and (1.3) the Navier-Stokes equations reduce to

$$
\begin{gathered}
\nabla \cdot(v \nabla v)=0 \\
\frac{\partial p}{\partial x_{1}}+h \frac{\partial h}{\partial x_{1}}=0 \\
\frac{\partial p}{\partial x_{2}}+h \frac{\partial h}{\partial x_{2}}=0 .
\end{gathered}
$$


In view of (1.1) and (1.2), the Ohm's law $\rho \boldsymbol{J}=\boldsymbol{E}+\boldsymbol{v} \times \mu_{0} \boldsymbol{H}$ gives

$$
\rho \boldsymbol{J}=\boldsymbol{E} .
$$

Moreover, since $\boldsymbol{E}$ is irrotational, we have, taking the curl of (5.1),

$$
\nabla \times(\rho \nabla \times \boldsymbol{H})=0
$$

and by (1.1)

$$
\nabla \cdot(\rho \nabla h)=0
$$

The energy equation reads

$$
-\nabla \cdot(\kappa \nabla u)=\boldsymbol{E} \cdot \boldsymbol{J}+v \sum_{i, k=1}^{3}\left(\frac{\partial v_{i}}{\partial x_{k}}+\frac{\partial v_{k}}{\partial x_{i}}\right) \frac{\partial v_{i}}{\partial x_{k}}
$$

where in the right hand side the first term represents the Joule heating and the second the viscous heating. Specializing (5.9) in our case we obtain, in view of (1.1), (1.2) and (5.6),

$$
-\nabla \cdot(\kappa \nabla u)=\rho|\nabla h|^{2}+v|\nabla v|^{2} .
$$

This equation, by (5.3) and (5.8), can also be written as follows

$$
\nabla \cdot[\kappa \nabla u+\rho h \nabla h+v v \nabla v]=0 .
$$

Once $h\left(x_{1}, x_{2}\right)$ is known as part of the solution of problem $(P)$, we can compute $p\left(x_{1}, x_{2}\right)$ from (5.4) and (5.5).

\section{REFERENCES}

[1] A. Ambrosetti - A. Malchiodi, Nonlinear Analysis and Semilinear Elliptic Problems, Cambridge University Press, Cambridge, 2007.

[2] F. CAFiero, Su un problema ai limiti relativo all'equazione $y^{\prime}=f(x, y, \lambda)$, Giornale di Matematiche di Battaglini, 77 (1947), pp. 28-35.

[3] F. CAFIERo, Sui problemi ai limiti relativi ad un'equazione differenziale ordinaria del primo ordine e dipendente da un parametro, Rend. Sem. Univ. Padova, 18 (1949), pp. 239-257.

[4] G. CimatTi, A plane problem of incompressible magnetohydrodynamics with viscosity and resistivity depending on the temperature, Rend. Mat. Acc. Lincei, 9 (2004), pp. 137-146.

[5] G. Cimatтi, Remark on existence, uniqueness and semi-explicit solvability of system of autonomous partial differential equations in diver- 
gence form with constant boundary conditions, to appear in Proc. (A) Roy. Soc. Edin.

[6] A. JefFreY, Magnetohydrodynamics, Oliver and Boyd LTD, John Wiley and Sons, Inc., New York 1966.

[7] K. ZaWISCHA, Über die Differentialgleichung $y^{\prime}=k f(x, y)$ deren Lösungkurve durch zwei gegebene Punkte hindurchgehen soll, Monatsh. für Math. und Phys. 37 (1930), pp. 103-124.

[8] G. ZWIRNER, Un criterio d'esistenza e unicità per gli integrali dell'equazione $y^{\prime}=\lambda f(x, y)$ passanti per due punti assegnati, Boll. Un. Mat. Ital., 3 (1948), pp. 15-18.

Manoscritto pervenuto in redazione il 25 gennaio 2011. 